\title{
Hypovitaminosis D in Geriatric Acute Care Unit: A Biomarker of Longer Length of Stay
}

\author{
Lise Hélard, ${ }^{1,2}$ Leyla Mateus-Hamdan, ${ }^{2,3}$ Olivier Beauchet, ${ }^{2,4}$ and Cédric Annweiler ${ }^{2,4,5}$ \\ ${ }^{1}$ Department of Geriatrics, Hospital of Laval, 53000 Laval, France \\ ${ }^{2}$ Division of Geriatric Medicine, Department of Neuroscience, Angers University Hospital, 49933 Angers Cedex 9, France \\ ${ }^{3}$ Department of Geriatrics, Hospital of Château-Gontier, 53200 Château-Gontier, France \\ ${ }^{4}$ UPRES EA 4638, Angers University, UNAM, 49000 Angers, France \\ ${ }^{5}$ Department of Medical Biophysics, Schulich School of Medicine and Dentistry, Robarts Research Institute, \\ The University of Western Ontario, London, ON, Canada N6A $5 \mathrm{~K} 8$ \\ Correspondence should be addressed to Cédric Annweiler; ceannweiler@chu-angers.fr
}

Received 9 June 2013; Revised 4 September 2013; Accepted 6 September 2013

Academic Editor: Paul Ashwood

Copyright @ 2013 Lise Hélard et al. This is an open access article distributed under the Creative Commons Attribution License, which permits unrestricted use, distribution, and reproduction in any medium, provided the original work is properly cited.

\begin{abstract}
Background. Hypovitaminosis D is linked to unstable health in older adults. Our objectives were to determine (i) the difference in length of stay (LOS) in geriatric acute care unit between inpatients with and without hypovitaminosis D and (ii) whether there was a linear association between serum 25-hydroxyvitamin D (25OHD) concentration and LOS. Methods. 253 inpatients admitted in 2008 to the geriatric acute care unit of Angers University Hospital, France, (mean age \pm standard deviation, $86.2 \pm 6.0$ years; $66.8 \%$ female) were included in this historical cohort study. LOS was calculated by subtracting day of admission from day of discharge. Hypovitaminosis D was defined as $25 \mathrm{OHD} \leq 50 \mathrm{nmol} / \mathrm{L}$ at the time of admission. Age, gender, place of life, functional independence, reason for admission, number of acute diseases and comorbidities, use of vitamin D supplements, and creatinine clearance were used as confounders. Results. Participants with hypovitaminosis D had longer LOS than their counterparts (15.2 \pm 8.2 days versus $12.1 \pm 7.0$ days, $P=0.017)$, underlining a mean difference of 3 days. 25OHD concentration inversely correlated $(r=-0.14, P=0.028)$ and was inversely associated with LOS (adjusted $\beta=-0.07$ [95\%CI: $-0.14 ;-0.02], P=0.043)$. Conclusions. We found an inverse linear association between serum 25OHD concentrations and LOS in a geriatric acute care unit. Participants with $25 \mathrm{OHD}>50 \mathrm{nmol} / \mathrm{L}$ were hospitalized on average 3 days less than those with $25 \mathrm{OHD} \leq 50 \mathrm{nmol} / \mathrm{L}$.
\end{abstract}

\section{Introduction}

Hypovitaminosis D is a highly prevalent condition, especially in older adults, with an estimated prevalence of 50-90\% depending on the definition used and the population studied $[1,2]$. Growing evidence has established that vitamin D exhibits not only skeletal effects but also a number of nonskeletal effects $[1,2]$. This finding explains why vitamin $D$ plays a much greater role in human health than previously thought and why lower 25-hydroxyvitamin D (25OHD) concentrations contribute to cellular dysfunction in many biological systems, which in turn is manifested by organ disorders and chronic diseases [1-4]. In particular, it has been reported, among older inpatients hospitalized in geriatric acute care units, that lower serum 25OHD concentrations at the time of admission are directly associated with a greater severity of chronic diseases [4] and higher risks of acute decompensation [5] and inhospital mortality [6]. In line with this, hypovitaminosis $\mathrm{D}$ has been proposed as a biomarker of longer length of stay (LOS) in acute care units [7-9]. For instance, lower serum 25OHD concentrations were independently associated with longer intensive care unit stay in cardiac surgical patients [7]. Hypovitaminosis D also doubled the risk of being hospitalized more than 14 days in a geriatric acute care unit [8] and, in another study, the combination of hypovitaminosis $\mathrm{D}$, male gender, and delirium predicted a 4.8-fold higher risk of longer LOS among geriatric inpatients [9]. Importantly, the average increase in LOS in geriatric acute care units attributable to hypovitaminosis D remains unknown. Moreover, while existing studies report 
an association between hypovitaminosis $\mathrm{D}$ and prolonged hospitalization, it remains unclear whether an inverse linear association exists over a range of $25 \mathrm{OHD}$ concentrations and whether any increase in $25 \mathrm{OHD}$ is associated with a decrease in LOS, regardless of the initial level.

We had the opportunity to examine these issues in a historical cohort of older inpatients hospitalized in a geriatric acute care unit. The objectives of this analysis were (i) to estimate the difference in hospitalization days between inpatients with and without hypovitaminosis $\mathrm{D}$ and (ii) to determine whether there was a linear association between serum 25OHD concentration and LOS.

\section{Materials and Methods}

2.1. Participants. The studied sample was a convenience of all inpatients consecutively admitted into the geriatric acute care unit of Angers University Hospital, France, between June and December 2008. Study inclusion criteria included unplanned admissions to hospital for people $\geq 75$ years and willingness to participate. Two hundred fifty-three inpatients met the study inclusion criteria and agreed to participate. Power analysis determined that 253 participants were sufficient to find significant correlations between serum 25OHD concentration and LOS with $77 \%$ power at $P<0.05$ [10]. The study was conducted in accordance with the ethical standards set forth in the Helsinki Declaration (1983). The entire study protocol was approved by the local Ethical Committee.

2.2. Outcome Measures. The LOS was calculated in number of days using the administrative registry of Angers University Hospital by subtracting day of admission from day of discharge.

The age, gender, usual place of life, degree of functional independence, main reason for admission, number of acute diseases and comorbidities, use of vitamin $\mathrm{D}$ supplements, and creatinine clearance were assessed as part of a standardized comprehensive geriatric assessment on admission to hospital and were used as potential confounders. Functional independence was measured from 0 to 6 (best) with the Autonomy, Gerontology, Iso-Resources Groups (AGGIR) model [11]. The usual place of life was also sought (i.e., home living versus institution dwelling defined as living in nursing home or in senior housing facilities). The main reasons for admission were separated into 6 categories: gait disorders/falls/orthopedic reasons, cardiovascular disorders, respiratory disorders, digestive disorders, neuropsychiatric disorders, and social issues. Acute diseases were defined as diseases with sudden onset and rapid progression, whatever their nature or site [5]. Comorbidities were diseases lasting at least 3 months or running a course with minimal change, whatever their nature or site [4]. Vitamin D supplementation was systematically noted from the primary care physician's prescription and sought by questioning the patient, whatever the dosage schedule or route of administration and regardless of the date of commencement.

Fasting early morning venous blood was collected from resting participants on the first day of hospital stay (i.e., the same day as the standardized anamnesis and physical examination) for the measurement of serum 25OHD and creatinine. Serum 25OHD concentration was measured by radioimmunoassay (DiaSorin Inc., Stillwater, $\mathrm{MN}$ ) with intra- and interassay precisions of $5.2 \%$ and $11.3 \%$, respectively. Hypovitaminosis $\mathrm{D}$ was defined as serum $25 \mathrm{OHD} \leq$ $50 \mathrm{nmol} / \mathrm{L}$ (to convert to $\mathrm{ng} / \mathrm{mL}$, divide by 2.496 ) according to the definition of the World Health Organization [12] and the Institute of Medicine [13]. Creatinine clearance was estimated using Cockcroft-Gault formula $\left(\left[\left(140-\right.\right.\right.$ age $\left._{\text {years }}\right) \times$ weight $_{\mathrm{kg}} /$ creatinine $\left._{\mu \mathrm{mol} / \mathrm{L}}\right] \times 1.04$ for females and $\times 1.25$ for males).

2.3. Statistics. Participants' characteristics were summarized using means and standard deviations or frequencies and percentages, as appropriate. As the number of observations was higher than 40 , comparisons were not affected by the shape of the error distribution and no transform was applied. First, comparisons between participants separated into two groups based on hypovitaminosis D were performed using Chi-square test or Student's $t$-test, as appropriate. Second, univariate and multiple (i.e., fully adjusted direct regression and backward regression) linear regressions were used to examine the association between LOS in geriatric acute care unit (dependent variable) and serum 25OHD concentration (independent variable), while adjusting for potential confounders. Third, a Pearson correlation was used to examine the relationship between serum $25 \mathrm{OHD}$ concentration and LOS in the unit. $P$ values $<0.05$ were considered significant. All statistics were performed using SPSS (v19.0, IBM Corporation, Chicago, IL).

\section{Results}

Among 253 inpatients included in this analysis (mean age \pm standard deviation $86.2 \pm 6.0$ years; $66.8 \%$ female; $100 \%$ Caucasian), the mean serum $25 \mathrm{OHD}$ concentration was $33.9 \pm 21.1 \mathrm{nmol} / \mathrm{L}$ and the mean LOS was $14.7 \pm 8.1$ days. We found that the LOS was longer among older inpatients with hypovitaminosis $\mathrm{D}$ compared to those with adequate vitamin $D$ status $(15.2 \pm 8.2$ days versus $12.1 \pm 7.0$ days, $P=0.017)$, underlining a mean LOS difference of 3.1 days between groups (Table 1). There were no other demographical and clinical differences, except for the use of vitamin D supplements (4.8\% in participants with hypovitaminosis D versus $38.9 \%$ in their counterparts, $P<0.001$ ).

Univariate and multiple linear regression models showed an inverse association between serum 25OHD concentration and LOS (unadjusted $\beta=-0.05$ [95\% confidence interval (CI): $-0.10 ;-0.01], P=0.028 ; \beta=-0.07$ [95\% CI: -0.14 ; -0.02 ], $P=0.043$ for adjusted model; $\beta=-0.08$ [95\% CI: $-0.14 ;-0.02], P=0.015$ for backward model). The creatinine clearance was also retained as an explanatory variable of LOS in the backward model ( $\beta=-0.02$ [95\% CI: $-0.05 ;-0.01$, $P=0.081$ ).

Finally, as illustrated in Figure 1, we found an inverse correlation between serum 25OHD concentration and LOS $(r=-0.14, P=0.028)$. 
TABLE 1: Characteristics and comparison of the participants $(n=253)$ separated into two groups based on hypovitaminosis D (i.e., serum $25 \mathrm{OHD} \leq 50 \mathrm{nmol} / \mathrm{L})$.

\begin{tabular}{|c|c|c|c|c|}
\hline & \multirow{2}{*}{ Total cohort $(n=253)$} & \multicolumn{2}{|c|}{ Serum $25 \mathrm{OHD}$ concentration } & \multirow{2}{*}{$P$ value ${ }^{*}$} \\
\hline & & $\leq 50(n=207)$ & $>50(n=46)$ & \\
\hline \multicolumn{5}{|l|}{ Demographical measures } \\
\hline Age (years), mean \pm SD & $86.2 \pm 6.0$ & $86.0 \pm 6.1$ & $86.8 \pm 5.3$ & 0.407 \\
\hline Female gender, $n(\%)$ & $169(66.8)$ & $134(64.7)$ & $35(76.1)$ & 0.139 \\
\hline $\operatorname{AGGIR}$ score $(/ 6)$, mean $\pm S D$ & $3.2 \pm 1.2$ & $3.2 \pm 1.3$ & $3.5 \pm 1.0$ & 0.308 \\
\hline Living at home, $n(\%)$ & $144(56.9)$ & $123(59.4)$ & $21(45.7)$ & 0.088 \\
\hline \multicolumn{5}{|l|}{ Clinical measures } \\
\hline Main reason for admission, $n(\%)$ & & & & 0.822 \\
\hline Gait disorders/falls/orthopaedic & $98(38.7)$ & $80(38.6)$ & $18(39.1)$ & \\
\hline Cardiovascular & $20(7.9)$ & $17(8.2)$ & $5(10.9)$ & \\
\hline Respiratory & $26(10.3)$ & $22(10.6)$ & $6(13.0)$ & \\
\hline Digestive & $17(6.7)$ & $11(5.3)$ & $0(0.0)$ & \\
\hline Neuropsychiatric & $47(18.6)$ & $40(19.3)$ & $8(17.4)$ & \\
\hline Social & $45(17.7)$ & $37(17.9)$ & $9(19.6)$ & \\
\hline Number of acute diseases, mean \pm SD & $3.0 \pm 1.4$ & $3.1 \pm 1.5$ & $2.6 \pm 1.1$ & 0.059 \\
\hline Number of comorbidities ${ }^{\dagger}$, mean \pm SD & $3.7 \pm 1.7$ & $3.7 \pm 1.7$ & $3.9 \pm 1.9$ & 0.487 \\
\hline Length of stay $(\text { days })^{\ddagger}$, mean \pm SD & $14.7 \pm 8.1$ & $15.2 \pm 8.2$ & $12.1 \pm 7.0$ & 0.017 \\
\hline Use of vitamin D supplements, $n(\%)$ & $26(10.3)$ & $10(4.8)$ & $17(36.9)$ & $<0.001$ \\
\hline \multicolumn{5}{|l|}{ Biological measures } \\
\hline Serum $25 \mathrm{OHD}$ concentration $(\mathrm{nmol} / \mathrm{L})$, mean \pm SD & $33.9 \pm 21.1$ & $25.1 \pm 7.3$ & $73.8 \pm 16.0$ & $<0.001$ \\
\hline Creatinine clearance $(\mathrm{mL} / \mathrm{min})^{\|}$, mean $\pm \mathrm{SD}$ & $56.4 \pm 42.8$ & $58.5 \pm 46.2$ & $46.3 \pm 16.7$ & 0.120 \\
\hline
\end{tabular}

25OHD: 25-hydroxyvitamin D; SD: standard deviation; AGGIR: Autonomy, Gerontology, Iso-Resources Groups.

${ }^{*}$ Comparisons based on the Chi-square test or Student's $t$-test, as appropriate.

${ }^{\dagger}$ Diseases lasting at least 3 months or running a course with minimal change.

${ }^{\ddagger}$ Number of hospitalization days in the geriatric acute care unit.

"Calculated from the Cockcroft formula $\left[\left(140\right.\right.$-age $\left.{ }_{\text {years }}\right) \times$ weight $_{\mathrm{kg}} /$ creatinine $\left._{\mu \mathrm{mol} / \mathrm{L}}\right] \times 1.04$ for females, and $\times 1.25$ for males.

$P$ significant (i.e., $<0.05)$ is indicated in bold.

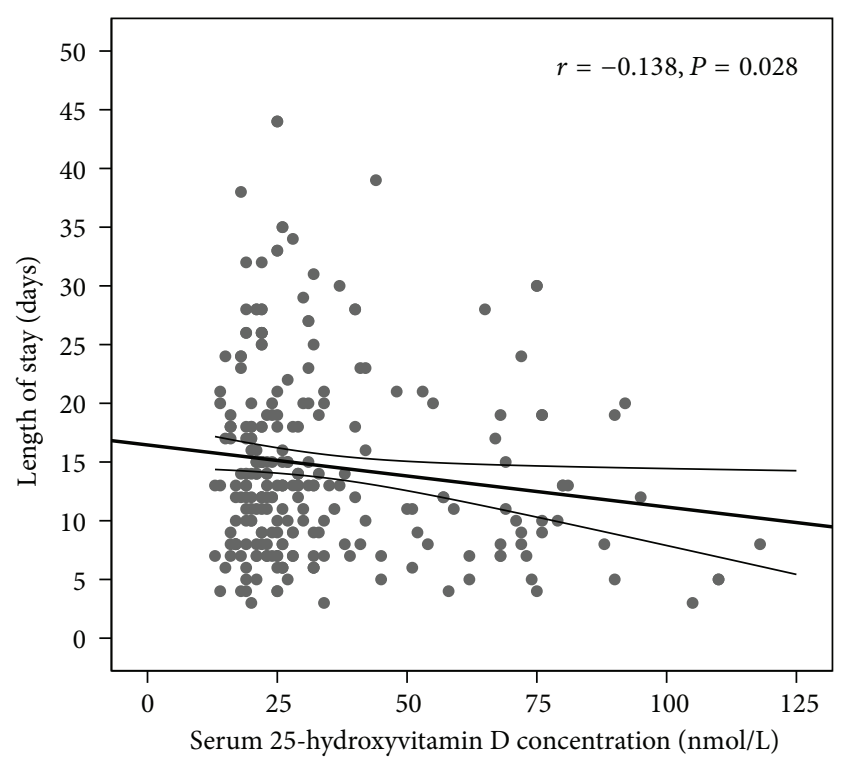

FIGURE 1: Relationship between the serum 25-hydroxyvitamin D concentration and the length of stay in the geriatric acute care unit. The thick line is the best-fit line, and the thin lines at the top and bottom are the limits of the $95 \%$ confidence interval.

\section{Discussion}

Our results show that, in a geriatric acute care unit, older inpatients with serum $25 \mathrm{OHD} \leq 50 \mathrm{nmol} / \mathrm{L}$ at time of admission had a LOS extended by approximately 3 days compared to those with $25 \mathrm{OHD}>50 \mathrm{nmol} / \mathrm{L}$. Additionally, we report the first evidence of a linear association between 25OHD concentration and LOS, suggesting that any increase in 25OHD was associated with a decrease in LOS whatever the initial vitamin D status.

Vitamin D is a secosteroid hormone that exhibits multiple biological actions mediated by the vitamin $\mathrm{D}$ receptor present in many cells and target tissues [1-3]. Since vitamin D is necessary for the regulation of cellular growth, differentiation, and function [3], lower vitamin D status leads to multiple organ dysfunction, disability, and unstable health status, which are all causes of deconditioning, polypharmacy, and longer LOS [14-16]. Consistently, previous literature from our group has provided compelling evidence that hypovitaminosis $\mathrm{D}$ could be regarded as a biomarker of agerelated disease burden $[4,5]$ and consequent longer LOS [7-9]. For instance, it has been reported that the risk of being in the highest tertile of LOS was multiplied by approximately 2 in case of hypovitaminosis D at baseline among 
geriatric inpatients [8]. However, the number of additional hospitalization days in case of hypovitaminosis D has not been examined yet. Thus, the results of the current study provide extra information, specifically, that older inpatients with hypovitaminosis $\mathrm{D}$ were hospitalized 3 days more in our geriatric acute care unit than those with adequate vitamin D status; that is, they had a 21 percent longer LOS (Table 1). The clinical relevance is that longer LOS exposes older inpatients to greater risks of disability, nosocomial infections, deconditioning, and higher healthcare costs $[16,17]$. Consequently, any decrease in LOS is beneficial to older inpatients. In the current study, we found a 0.14-day decrease in LOS per $1 \mathrm{nmol} / \mathrm{L}$ increase in serum 25OHD concentration (Figure 1), that is, a 1-day decrease in LOS per $7.1 \mathrm{nmol} / \mathrm{L}$ increase in 25OHD. It has been previously reported that vitamin D supplementation produces an elevation of serum $25 \mathrm{OHD}$ concentration of approximately $2.5 \mathrm{nmol} / \mathrm{L}$ per $100 \mathrm{IU} /$ day of supplements [18]. It can thus be inferred that each additional $300 \mathrm{IU}$ vitamin D per day is associated with a 1-day shortening of LOS among geriatric inpatients. Such estimates may help to justify, plan, evaluate, and compare the effectiveness of interventions aiming at shortening the LOS in geriatric acute care units with vitamin D supplements.

Some potential limitations of our study should be considered. Firstly, the study cohort was restricted to Caucasian older inpatients hospitalized in a single geriatric acute care unit who might be unrepresentative of all older inpatients. Secondly, the use of an observational and retrospective design prevented inferring causality. Thirdly, although we were able to control for many important characteristics that could bias the results, residual prehospital health conditions influencing both vitamin D status and LOS, such as the body mass index, were not considered. Finally, it has to be kept in mind that, in some cases, LOS does not only depend on bioclinical aspects but is influenced by other factors, including number of beds available, human resource employees, and health insurance guidelines [15], which could not be taken into account here.

\section{Conclusions}

Increased serum 25OHD concentrations were linearly associated with shorter LOS in this cohort of acute care geriatric inpatients. Those with an adequate vitamin $\mathrm{D}$ status were hospitalized 3 days less than those with hypovitaminosis D. Our findings reinforce the conceptualization of vitamin $\mathrm{D}$ as a biomarker of the health status of inpatients in geriatric acute care unit and provide an additional rationale for prescribing vitamin D supplements in this population. Multicenter randomized controlled trials are required to investigate the benefits of vitamin D supplementation on LOS with higher levels of evidence.

\section{Conflict of Interests}

Professor Beauchet has served as an unpaid consultant for Ipsen Pharma company and serves as an associate editor for Gériatrie, Psychologie et Neuropsychiatrie du Vieillissement. He has no relevant financial interest in this paper.
Dr. Annweiler has served as an unpaid consultant for Ipsen Pharma company and serves as an associate editor for Gériatrie, Psychologie et Neuropsychiatrie du Vieillissement and for the Journal of Alzheimer's Disease. He has no relevant financial interest in this paper.

\section{Authors' Contribution}

Annweiler has full access to the data in the study and takes responsibility for the integrity of the data and the accuracy of the data analyses. Study concept and design were done by Annweiler. Acquisition of data was done by Hélard. Analysis and interpretation of data were done by Hélard and Annweiler. Drafting of the paper was done by Hélard and Annweiler. Critical revision of the paper for important intellectual content was done by Beauchet and MateusHamdan. Obtained funding: not applicable. Annweiler has the statistical expertise. Annweiler was responsible for the administrative, technical, or material support. Study supervision was done by Annweiler.

\section{Acknowledgments}

The authors are grateful to the participants for their cooperation. The authors also thank Melinda Beaudenon, MS, Jennifer Gautier, BS, and Samuel Thiery, BS, from Angers University Hospital, France, for daily assistance.

\section{References}

[1] M. F. Holick, "Vitamin D deficiency," The New England Journal of Medicine, vol. 357, no. 3, pp. 266-281, 2007.

[2] C. Annweiler, J.-C. Souberbielle, A.-M. Schott, L. de Decker, G. Berrut, and O. Beauchet, "Vitamin D in the elderly: 5 points to remember," Geriatrie et Psychologie Neuropsychiatrie du Vieillissement, vol. 9, no. 3, pp. 259-267, 2011.

[3] M. Peterlik and H. S. Cross, "Vitamin D and calcium insufficiency-related chronic diseases: molecular and cellular pathophysiology," European Journal of Clinical Nutrition, vol. 63, no. 12, pp. 1377-1386, 2009.

[4] O. Beauchet, L. Hélard, M. Montero-Odasso, L. de Decker, G. Berrut, and C. Annweiler, "Hypovitaminosis D in geriatric inpatients: a marker of severity of chronic diseases," Aging Clinical and Experimental Research, vol. 24, pp. 188-192, 2012.

[5] A. Sutra del Galy, M. Bertrand, F. Bigot et al., "Vitamin D insufficiency and acute care in geriatric inpatients," Journal of the American Geriatrics Society, vol. 57, no. 9, pp. 1721-1723, 2009.

[6] C. Annweiler, S. Pochic, B. Fantino et al., "Serum vitamin $\mathrm{D}$ concentration and short-term mortality among geriatric inpatients in acute care settings," Advances in Therapy, vol. 27, no. 4, pp. 245-249, 2010.

[7] A. Zittermann, J. Kuhn, J. Dreier, C. Knabbe, J. F. Gummert, and J. Börgermann, "Vitamin D status and the risk of major adverse cardiac and cerebrovascular events in cardiac surgery," European Heart Journal, vol. 34, pp. 1358-1364, 2013.

[8] O. Beauchet, C. P. Launay, F. Maunoury, L. de Decker, B. Fantino, and C. Annweiler, "Association between vitamin D deficiency and long hospital stay in geriatric acute care unit: 
results from a pilot cohort study," Aging Clinical and Experimental Research, vol. 25, no. 1, pp. 107-109, 2013.

[9] O. Beauchet, C. Launay, L. de Decker, B. Fantino, A. Kabeshova, and C. Annweiler, "Who is at risk of long hospital stay among patients admitted to geriatric acute care unit? Results from a prospective cohort study," The Journal of Nutrition, Health \& Aging, vol. 17, no. 8, pp. 695-699, 2013.

[10] D. Machin, M. Campbell, P. Fayers, and A. Pinol, Sample Size Tables for Clinical Studies, Blackwell Science, Oxford, UK, 2nd edition, 1997.

[11] J. M. Vetel, R. Leroux, and J. M. Ducoudray, "AGGIR. Practical use. Geriatric Autonomy Group Resources Needs," Soins. Gérontologie, no. 13, pp. 23-27, 1998.

[12] World Health Organization, Prevention and Management of Osteoporosis: Report of a WHO Scientific Group, World Health Organization, Geneva, Switzerland, 2003.

[13] A. C. Ross, J. E. Manson, S. A. Abrams et al., "The 2011 report on dietary reference intakes for calcium and vitamin $\mathrm{D}$ from the Institute of Medicine: what clinicians need to know," Journal of Clinical Endocrinology and Metabolism, vol. 96, no. 1, pp. 53-58, 2011.

[14] C. Subramaniam, L. C. Gray, and S. Farish, "Predictors of length of stay in a geriatric assessment and rehabilitation unit," Australian Health Review, vol. 18, no. 2, pp. 56-68, 1995.

[15] W. Chung, S.-M. Oh, T. Suh, Y. M. Lee, B. H. Oh, and C.-W. Yoon, "Determinants of length of stay for psychiatric inpatients: analysis of a national database covering the entire Korean elderly population," Health Policy, vol. 94, no. 2, pp. 120-128, 2010.

[16] M. Zanocchi, B. Maero, P. Maina et al., "Factors predicting a prolonged hospital stay in elderly patients," Minerva Medica, vol. 93, no. 2, pp. 135-143, 2002.

[17] R. M. Palmer, "Acute hospital care of the elderly: minimizing the risk of functional decline," Cleveland Clinic Journal of Medicine, vol. 62 , no. 2, pp. 117-128, 1995.

[18] R. P. Heaney, "Vitamin D in health and disease," Clinical Journal of the American Society of Nephrology, vol. 3, no. 5, pp. 1535-1541, 2008. 


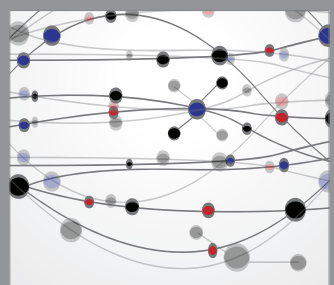

The Scientific World Journal
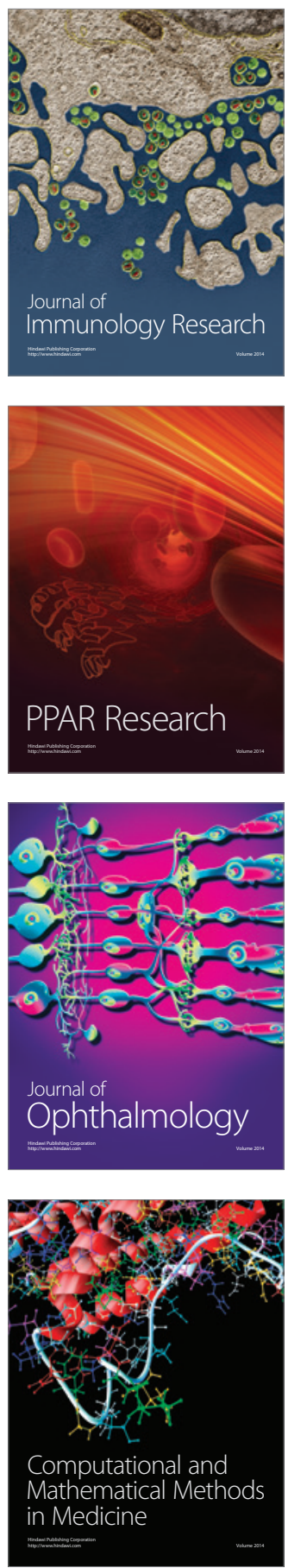

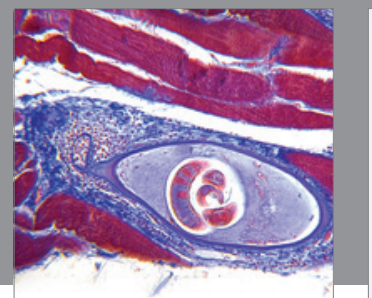

Gastroenterology

Research and Practice
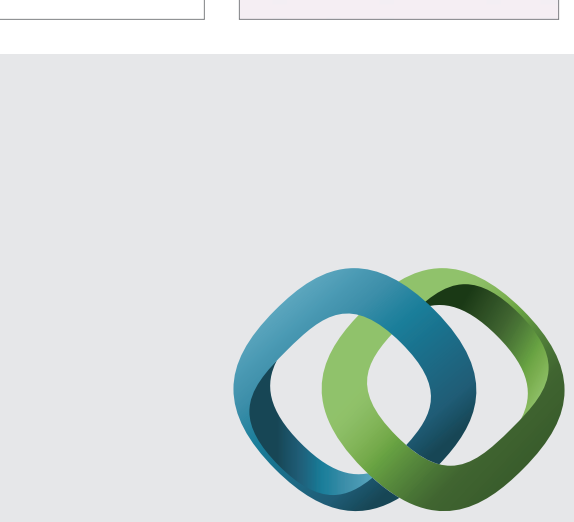

\section{Hindawi}

Submit your manuscripts at

http://www.hindawi.com
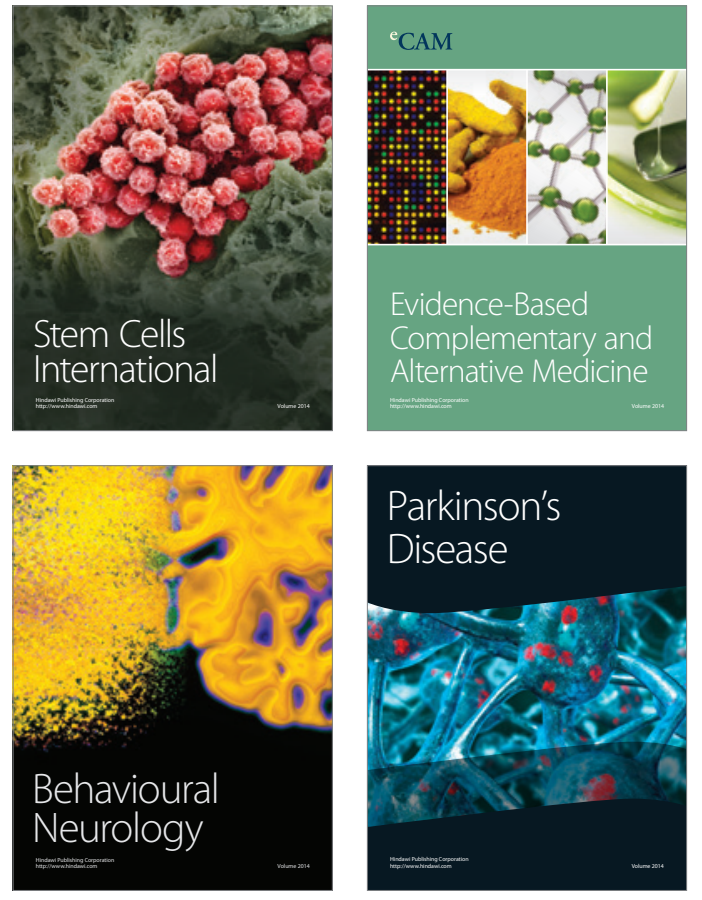
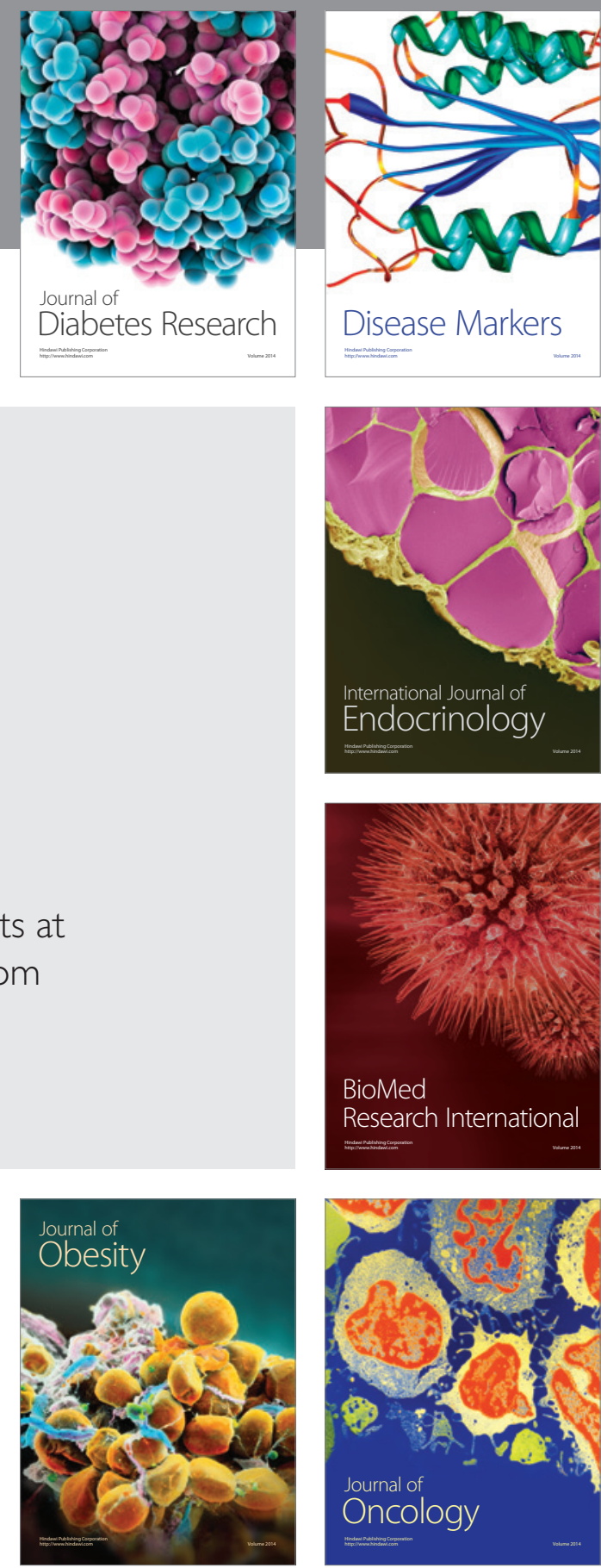

Disease Markers
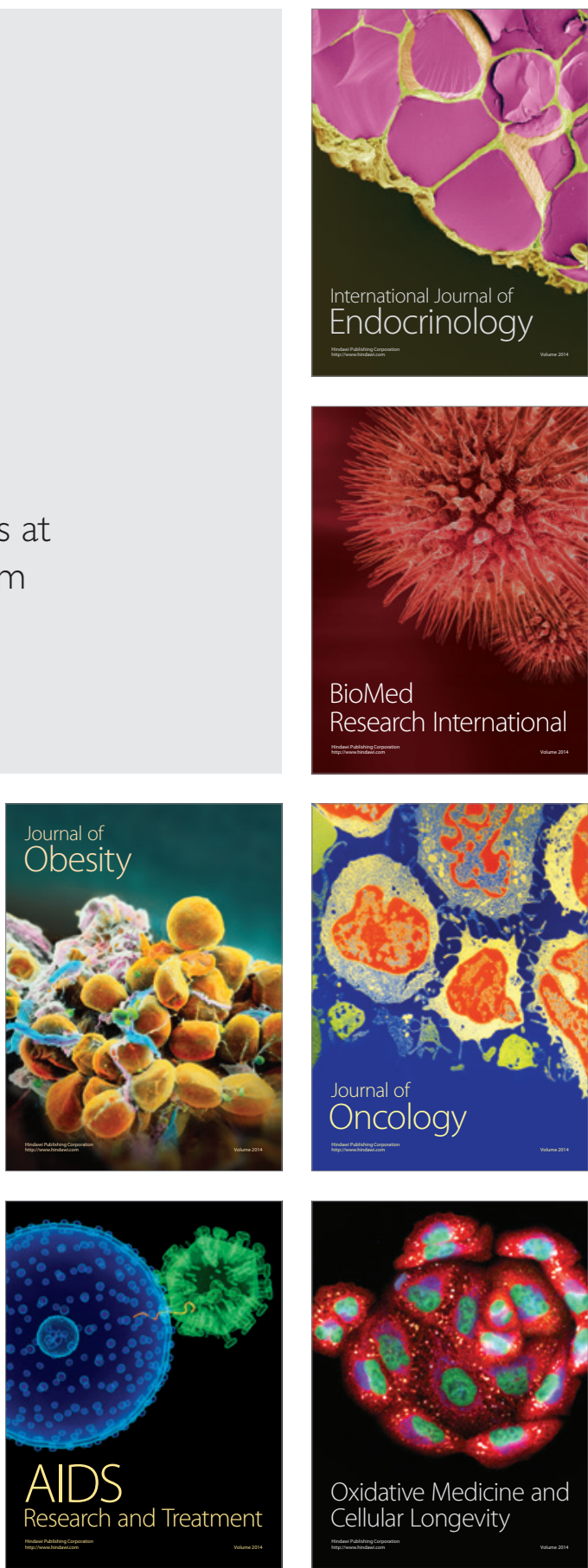